\title{
Trends in repair of intact and ruptured descending thoracic aortic aneurysms in the United States: A population-based analysis
}

\author{
Arman Kilic, MD, ${ }^{\mathrm{a}}$ Ashish S. Shah, MD, ${ }^{\mathrm{a}}$ James H. Black III, MD, ${ }^{\mathrm{a}}$ Glenn J. R. Whitman, MD, ${ }^{\mathrm{a}}$ \\ David D. Yuh, MD, ${ }^{\mathrm{b}}$ Duke E. Cameron, MD, ${ }^{\mathrm{a}}$ and John V. Conte, $\mathrm{MD}^{\mathrm{a}}$
}

\begin{abstract}
Background: To evaluate trends and outcomes of descending thoracic aortic aneurysm (DTAA) repair in the United States.

Methods: Adults undergoing DTAA repair between 1998 and 2008 were identified in the Nationwide Inpatient Sample. To limit confounding, patients with connective tissue disorders, aortic dissection, or thoracoabdominal aneurysms were excluded. Stratification was based on intact versus ruptured DTAA and open versus endovascular approach. Standardized annual rates of repair were calculated based on US Census Bureau population estimates. Logistic regression analysis incorporating multiple patient, operative, and hospital variables was used for risk adjustment.
\end{abstract}

Results: A total of 20,568 DTAA patients (intact, 17,780; ruptured, 2788) underwent repair (open, 15,265; endovascular, 5303). Patients undergoing repair in the more recent era had higher comorbidity burdens than those undergoing repair in the earlier era. Despite this, annual rates of repair for both intact and ruptured DTAAs increased significantly during the study period (intact, 2.2-10.6 per 1 million; ruptured, 0.8-1.3 per 1 million; $P<.05)$, primarily because of increases in rates of endovascular repair in recent years. Operative mortality decreased from $10.3 \%$ to $3.1 \%$ for repairs of intact DTAAs $(P<.001)$ and from $52.6 \%$ to $23.4 \%$ for ruptured DTAAs $(P=.002)$. Endovascular repair was associated with reduced risk-adjusted mortality for both intact (odds ratio, $0.31 ; P<.001$ ) and ruptured (odds ratio, $0.41 ; P=.001$ ) DTAAs.

Conclusions: Although patients undergoing DTAA repair in the modern era have a higher comorbidity burden, rates of repair have increased and operative mortality has decreased, in part because of the increasing adoption of endovascular approaches. (J Thorac Cardiovasc Surg 2014;147:1855-60)

Thoracic aortic aneurysms have an estimated incidence of 6 to 10 per 100,000 person-years, of which $40 \%$ are composed of descending thoracic aortic aneurysms (DTAAs). ${ }^{1-3}$ As with other aortic pathologic conditions, the management of DTAA has changed significantly during the past several years. For instance, the evolution of multidetector computed tomography has resulted in more rapid image acquisition and improved 3-dimensional reconstruction. This has led to better evaluation of the complex anatomic features in DTAA, including aortic tortuosity, relation of the aneurysm to branch vessels, and presence of dissection. ${ }^{4}$ Operative techniques have also changed, including the adoption of

From the Division of Cardiac Surgery, ${ }^{\mathrm{a}}$ Department of Surgery, The Johns Hopkins Medical Institutions, Baltimore, Md; and the Section of Cardiac Surgery, ${ }^{\mathrm{b}}$ Department of Surgery, Yale University School of Medicine, New Haven, Conn.

Supported by departmental funds from the Department of Surgery, The Johns Hopkins Hospital.

Disclosures: Authors have nothing to disclose with regard to commercial support.

Presented at the Annual Scientific Sessions of the American Heart Association, Los Angeles, Calif, November 3-7, 2012

Received for publication Feb 11, 2013; revisions received May 2, 2013; accepted for publication June 27, 2013; available ahead of print Aug 29, 2013.

Address for reprints: John V. Conte, MD, Division of Cardiac Surgery, Department of Surgery, The Johns Hopkins Hospital, 1800 Orleans St, Ste 7107, Baltimore, MD

21287 (E-mail: jconte@jhmi.edu).

$0022-5223 / \$ 36.00$

Copyright (C) 2014 Published by Elsevier Inc. on behalf of The American Association for Thoracic Surgery

http://dx.doi.org/10.1016/j.jtcvs.2013.06.032 organ-specific protective strategies, such as cerebrospinal fluid drainage, reimplantation of intercostal arteries, regional hypothermia, use of evoked-potential monitoring, and distal aortic perfusion, to reduce the risk of spinal cord ischemia. ${ }^{5-7}$

The introduction of thoracic endovascular aortic repair (TEVAR) is perhaps the most notable change in the management of DTAA. TEVAR is particularly attractive given the frequently significant comorbidity burden of these patients. The aim of this study was to evaluate trends and outcomes of DTAA repair in the United States during the past several years.

\section{METHODS \\ Data Source}

The Nationwide Inpatient Sample (NIS) was used for this study. This registry was developed by the Agency for Healthcare Research and Quality, and contains inpatient data on more than 8 million patient visits per year, with more than 1050 participating hospitals. ${ }^{8}$ After weighting, this reflects approximately $95 \%$ of hospital discharges within the United States. The data provided by the NIS are deidentified. The Johns Hopkins Institutional Review Board, therefore, granted this study exempt status.

\section{Study Population}

Adults ( $>17$ years) undergoing DTAA repair between January 1998 and December 2008 were identified in the NIS database. International Classification of Diseases, Ninth Revision (ICD-9), codes were used to identify the study population. Intact thoracic aneurysms were identified with the International Classification of Diseases, Ninth Revision (ICD-9), diagnosis 


\section{Abbreviations and Acronyms}

COPD = chronic obstructive pulmonary disease

DTAA $=$ descending thoracic aortic aneurysm

NIS = Nationwide Inpatient Sample

TEVAR $=$ thoracic endovascular aortic repair

code 441.2, and ruptured thoracic aneurysms were identified with the code 441.1. Procedure codes 38.45 and 39.73 were used to identify open and endovascular repairs, respectively. Patients with procedure codes for both open and endovascular DTAA repair were excluded from the study. To isolate DTAAs, patients with aortic dissections (diagnosis codes 441.00441.03), thoracoabdominal aneurysms (diagnosis codes 441.3-441.9 or procedure code 38.44 or 39.71 ), abdominal aortic aneurysms (diagnosis code 441.3 or 441.4 or procedure code 38.46 or 39.71 ), or connective tissue disorders (diagnosis codes 446.0-446.7 and 759.82) were excluded from the analysis. In addition, patients undergoing cardiac valve or coronary bypass surgery (procedure codes 35.11, 35.12, 35.14, 35.21, 35.22, $35.23,35.24,35.27,35.28$, and $36.10-36.19$ ), aortic to subclavian or aortic to carotid bypass (39.22), intrathoracic bypass (39.23), or hypothermic circulatory arrest (procedure code 39.62 ) were excluded.

\section{Data Analysis}

Stratification was based on intact versus ruptured DTAA repair and open versus endovascular approach. Baseline characteristics were initially evaluated for the study cohort. These included patient-specific variables, such as age, sex, and comorbidities, including prior myocardial infarction, congestive heart failure, cerebrovascular disease, chronic obstructive pulmonary disease (COPD), diabetes mellitus, liver disease, and chronic renal insufficiency. The Charlson comorbidity index, which is a validated measure of overall comorbidity burden, was also calculated for each patient. ${ }^{9}$ We evaluated hospital-specific variables, including teaching status, rural versus urban location, and large size designation, as determined by number of beds. These baseline characteristics were compared between patients undergoing repair in the pre-TEVAR (1998-2004) versus TEVAR (20052008) era.

Annual rates of repair were also calculated for both intact and ruptured cases. These were based on population estimates as provided by the US Census Bureau. ${ }^{10}$ In addition to evaluating overall rates of repair, we also examined rates of open versus endovascular repair. Annual unadjusted operative mortality rates were then calculated for the intact and ruptured cohorts. Linear regression was used to determine the statistical significance of the trend in operative mortality during the study time period.

Finally, multivariable logistic regression models were constructed to identify predictors of operative mortality. Separate models were created for intact and ruptured DTAA repairs. Variables included in the model were age, sex, comorbidities, teaching status of hospital, hospital location, hospital size, era, and operative approach.

Survey statistics were used for statistical comparisons. This included the adjusted Wald test for continuous data and a design-based Pearson $\chi^{2}$ test for categorical data. Odds ratios from the multivariable model were presented with $95 \%$ confidence intervals. All statistical analyses were performed with Stata software, version 11 (StataCorp LP, College Station, Tex).

\section{RESULTS}

\section{Overall Study Cohort}

A total of 20,568 patients underwent DTAA repair during the study period. The mean age for the overall cohort was $64.7 \pm 14.0$ years. There were $5594(27.2 \%)$ patients aged 75 years or older. Most patients in the study were male $(58.2 \% ; \mathrm{n}=11,971)$. The most common comorbidity was COPD $(24.7 \% ; \mathrm{n}=5080)$, followed by congestive heart failure $(11.1 \% ; \mathrm{n}=2283)$ and cerebrovascular disease $(10.9 \% ; \mathrm{n}=2242)$. Most repairs were performed at large $(81.6 \% ; \mathrm{n}=16,783)$ teaching hospitals $(78.9 \%$; $\mathrm{n}=16,228)$ located in an urban setting $(98.7 \% ; \mathrm{n}=20,301)$.

\section{Trends in Baseline Characteristics Over Time}

Several significant differences were noted when comparing characteristics of patients undergoing repair of intact DTAA in the pre-TEVAR versus TEVAR era. Patients undergoing repair of intact DTAAs in the TEVAR era were older and had more comorbidities (Table 1). More specifically, those in the TEVAR era had higher rates of prior myocardial infarction, diabetes mellitus, and chronic renal insufficiency, as well as a higher overall Charlson comorbidity index. There was also a trend toward a higher rate of COPD in the TEVAR era. Hospital characteristics were comparable between eras.

Similarly, patients undergoing repair of ruptured DTAAs in the TEVAR era had a higher overall comorbidity burden, as measured by the Charlson score, compared with those undergoing repair of ruptured DTAAs in the pre-TEVAR era (Table 2). Chronic renal insufficiency was approximately 2-fold more common in the TEVAR era. Similar to repairs of intact DTAAs, hospital characteristics were similar between eras for the repairs of ruptured DTAAs.

\section{Rates of Repair}

The overall repair rates of intact DTAA increased significantly during the study period, from 2.2 to 10.6 per 1 million in 1998 and 2008, respectively $(r=0.89, P<.001)$ (Figure 1 , $A)$. Although open repair rates for intact DTAAs remained relatively stagnant during the past few years of the study period (open, from 3.8 in 2005 to 4.4 in 2008 per 1 million), the introduction of endovascular approaches in 2005 and their increasing use thereafter (TEVAR, from 1.0 in 2005 to 6.2 in 2008 per 1 million) resulted in increased overall rates.

The repair rates of ruptured DTAA also increased significantly during the study period, although to a less significant degree as that observed with the intact cohort, from 0.8 to 1.3 per 1 million $(r=0.59, P<.001)$ (Figure $1, B)$. Similar to the intact group, open repair rates for ruptured DTAAs were relatively stagnant in recent years (open, from 0.6 in 2005 to 0.6 in 2008 per 1 million), with the increasing adoption of endovascular therapy (TEVAR, from 0.1 in 2005 to 0.7 in 2008 per 1 million) driving the increase in overall repair rates.

\section{Operative Mortality}

Unadjusted operative mortality rates were significantly lower with TEVAR versus open surgery for both the intact (TEVAR vs open, $2.7 \%$ vs $6.3 \% ; P<.001$ ) and ruptured 
TABLE 1. Study population characteristics of the cohort undergoing repair for intact DTAAs

\begin{tabular}{|c|c|c|c|c|}
\hline \multirow[b]{2}{*}{ Variables } & \multirow[b]{2}{*}{ Repair of intact DTAA $(n=17,780)$} & \multicolumn{3}{|c|}{ Era } \\
\hline & & $1998-2004(n=8428)$ & $2005-2008(n=9352)$ & $P$ value \\
\hline Age, y & $64.1 \pm 14.1$ & $62.4 \pm 13.6$ & $65.6 \pm 14.3$ & $<.001$ \\
\hline Female sex & $7361(41)$ & $3540(42)$ & $3821(41)$ & .52 \\
\hline Charlson comorbidity index & $1.02 \pm 1.17$ & $0.83 \pm 0.97$ & $1.18 \pm 1.32$ & $<.001$ \\
\hline \multicolumn{5}{|l|}{ Comorbidities } \\
\hline Myocardial infarction & $1034(6)$ & $402(5)$ & $632(7)$ & .03 \\
\hline Congestive heart failure & $1866(10)$ & $818(10)$ & $1048(11)$ & .21 \\
\hline Cerebrovascular disease & $1776(10)$ & $856(10)$ & $920(10)$ & .76 \\
\hline COPD & $4384(25)$ & $1938(23)$ & $2446(26)$ & .07 \\
\hline Diabetes mellitus & $1789(10)$ & $659(8)$ & $1130(12)$ & $<.001$ \\
\hline Liver disease & $97(0.5)$ & $37(0.4)$ & $60(0.6)$ & .41 \\
\hline Chronic renal insufficiency & $1185(7)$ & $249(3)$ & $936(10)$ & $<.001$ \\
\hline Teaching hospital & $13,986(79)$ & $6777(80)$ & $7209(77)$ & .51 \\
\hline Urban hospital location & $17,529(99)$ & $8329(99)$ & $9200(98)$ & .59 \\
\hline Large hospital & $14,611(82)$ & $7129(85)$ & $7482(80)$ & .28 \\
\hline
\end{tabular}

Data are given as mean \pm SD or number $(\%)$. DTAA, Descending thoracic aortic aneurysm; COPD, chronic obstructive pulmonary disease.

(TEVAR vs open, $21.5 \%$ vs $36.6 \% ; P<.001$ ) cohorts. When grouped together, overall unadjusted operative mortality rates decreased significantly during the study period for repairs of both intact and ruptured DTAAs. For repairs of intact DTAAs, operative mortality decreased from $10.3 \%$ in 1998 to $3.1 \%$ in $2008(P<.001)$ (Figure 2, A). For repairs of ruptured DTAAs, operative mortality decreased from $52.6 \%$ in 1998 to $23.4 \%$ in $2008(P=.002)$ (Figure 2, $B)$.

\section{Predictors of Operative Mortality}

There were several significant predictors of operative mortality identified in the intact DTAA cohort. These included increasing age, with each additional decade of age being associated with an incremental 50\% increase in operative mortality (Table 3 ). Cerebrovascular and liver diseases were each associated with a more than 4-fold increase in operative mortality risk. Prior myocardial infarction and chronic renal insufficiency each also exerted a nearly 2-fold increase in operative mortality risk. Those repaired with an open approach were roughly 3 times more likely to die than those undergoing TEVAR. Earlier era was also a significant risk factor for operative mortality.

Increasing age increased the risk of operative mortality in the ruptured cohort as well (Table 4). Each decade increase in age was associated with a $30 \%$ increase in mortality risk. Comorbidities and teaching characteristics did not affect mortality in the ruptured cases. An open approach was associated with a 2.7 -fold increase in mortality risk compared with TEVAR in ruptured DTAAs. The effect of earlier era on mortality risk observed in unadjusted analysis no longer persisted in the risk-adjusted model.

Because of the greater number of patients in the intact DTAA cohort, the multivariable model for that particular

TABLE 2. Study population characteristics of the cohort undergoing repair of ruptured DTAAs

\begin{tabular}{|c|c|c|c|c|}
\hline \multirow[b]{2}{*}{ Variables } & \multirow[b]{2}{*}{ Repair of ruptured DTAAs $(n=2788)$} & \multicolumn{3}{|c|}{ Era } \\
\hline & & $1998-2004(n=1596)$ & $2005-2008(n=1192)$ & $P$ value \\
\hline Age, y & $68.6 \pm 13.4$ & $68.0 \pm 13.1$ & $69.4 \pm 13.8$ & .23 \\
\hline Female sex & $1228(44)$ & $660(41)$ & $568(48)$ & .17 \\
\hline Charlson comorbidity index & $2.14 \pm 1.26$ & $2.01 \pm 1.14$ & $2.32 \pm 1.40$ & .007 \\
\hline \multicolumn{5}{|l|}{ Comorbidities } \\
\hline Myocardial infarction & $253(9)$ & $140(9)$ & $113(9)$ & .82 \\
\hline Congestive heart failure & $428(15)$ & $248(16)$ & $180(15)$ & .90 \\
\hline Cerebrovascular disease & 466 (17) & 267 (17) & 199 (17) & .98 \\
\hline COPD & $695(25)$ & $399(25)$ & $296(25)$ & .98 \\
\hline Diabetes mellitus & $227(8)$ & $107(7)$ & $120(10)$ & .17 \\
\hline Liver disease & $11(0.4)$ & $0(0)$ & $11(0.9)$ & .09 \\
\hline Chronic renal insufficiency & $320(11)$ & $136(9)$ & $184(15)$ & .02 \\
\hline Teaching hospital & $2241(80)$ & $1300(81)$ & $941(79)$ & .60 \\
\hline Urban hospital location & 2777 (99) & $1590(99)$ & $1187(99)$ & .95 \\
\hline Large hospital & $2173(78)$ & $1224(77)$ & $949(80)$ & .57 \\
\hline
\end{tabular}

Data are given as mean \pm SD or number (\%). DTAA, Descending thoracic aortic aneurysm; COPD, chronic obstructive pulmonary disease. 

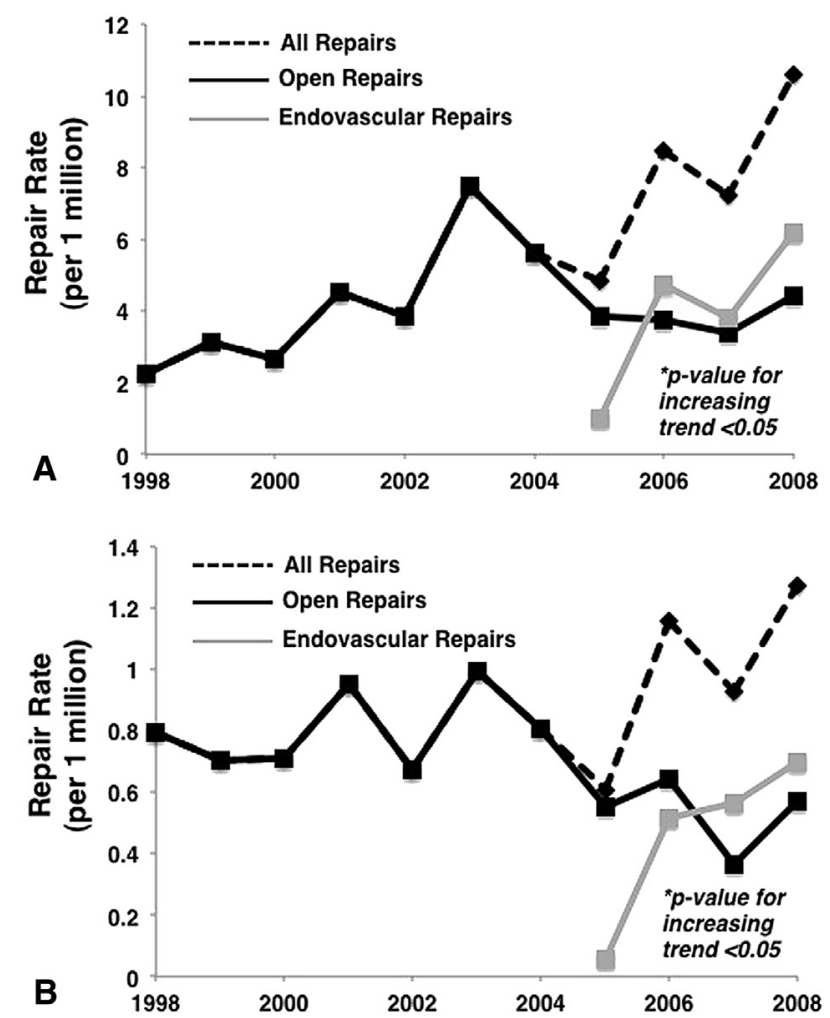

FIGURE 1. Standardized annual rates of open and endovascular repair of intact (A) and ruptured (B) descending thoracic aortic aneurysms.

analysis had higher predictive power. Moreover, the c-index for the model for repair of intact DTAAs was 0.80 . This was higher than the c-index for the multivariable model for repair of ruptured DTAAs, which was 0.66 .

\section{DISCUSSION \\ Study Findings}

In this study, we evaluated trends and outcomes of DTAA repair in the United States during the past decade. There were several important findings from this populationbased analysis. Foremost, standardized rates of repair had significantly increased over time for both intact and ruptured DTAAs. Given that the rates of open repair were relatively constant in the latter portion of the study period, the increasing adoption of TEVAR, starting in 2005, is the most likely driving factor for these increased rates of repair. Because patients in more recent years had higher comorbidity burdens, this suggests that TEVAR has helped augment the overall pool of DTAA repairs, in part by allowing sicker patients to undergo surgical intervention.

Furthermore, we found that operative mortality rates decreased significantly during the study period for repairs of both intact and ruptured DTAAs. Again, these findings occurred despite a higher comorbidity burden in patients. In multivariable analysis, we found that increasing age was a significant predictor of operative mortality for repairs
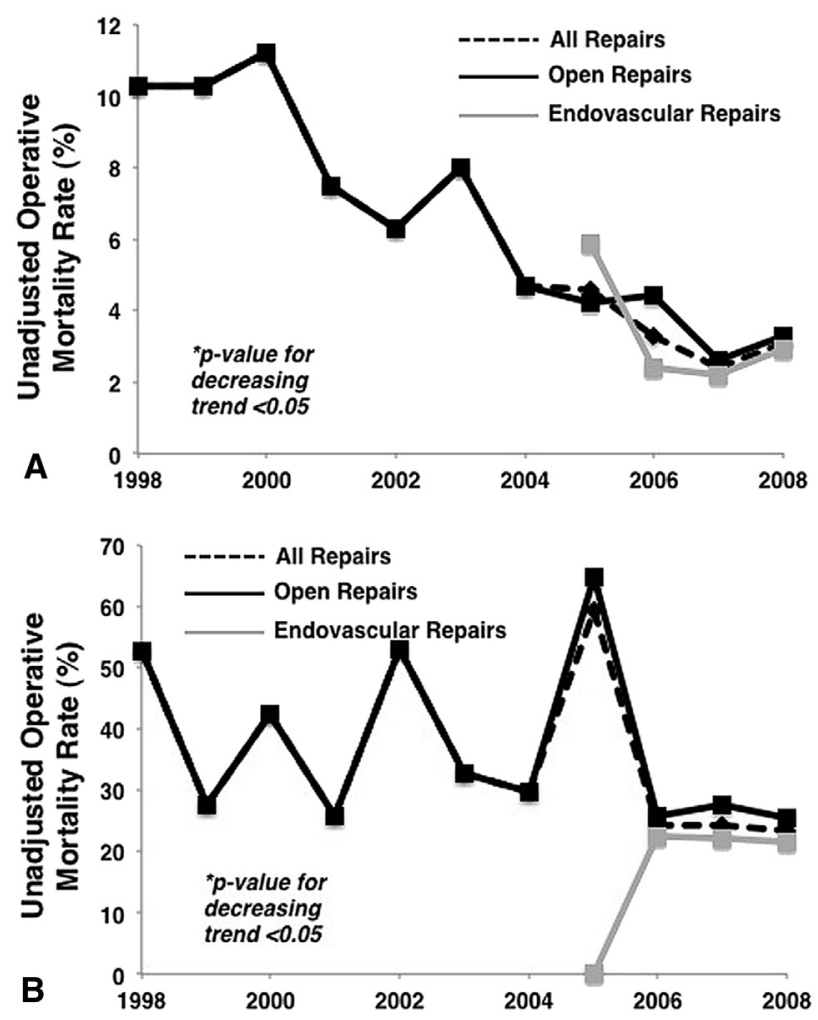

FIGURE 2. Annual unadjusted operative mortality rates for repair of intact (A) and ruptured (B) descending thoracic aortic aneurysms (DTAAs). In 2005, there were only 16 endovascular repairs of ruptured DTAAs.

of both intact and ruptured DTAAs. Older age is a wellknown predictor of adverse outcomes after major aortic procedures. A statewide analysis of thoracoabdominal aortic aneurysm repairs demonstrated steep increases in 1-year mortality with increasing age, from $18 \%$ for 50 - to 59 year-old patients to $40 \%$ for 80 - to 89 -year-old patients. ${ }^{11}$ A similar multicenter analysis performed for abdominal aortic aneurysms showed parallel results, with 1-year mortality ranging from $2.9 \%$ in those aged 51 to 60 years to $15 \%$ for those aged 81 to 90 years. ${ }^{12}$ Finally, in multivariable analysis, we found that, after accounting for patient and hospital characteristics, open surgical repair was associated with a significant increase in mortality risk compared with TEVAR for both intact and ruptured DTAAs.

\section{Prior Studies}

Indeed, open repair of DTAA has been associated with significant morbidity and mortality in prior series. In a single-institution review of 387 patients, the in-hospital mortality rate was $4.4 \%$, with a 30 -day mortality of $2.8 \%{ }^{13}$ In another single-institution study of 300 patients, the 30-day mortality was $8 \% .{ }^{7}$ An earlier study of 366 patients demonstrated an in-hospital mortality rate of $12 \%$ that decreased to $9.9 \%$ if ruptured aneurysms were excluded. ${ }^{14}$ These data generally coincide with our current 
TABLE 3. Multivariable analysis for inpatient mortality after repair of intact DTAAs

\begin{tabular}{lcc}
\hline \multicolumn{1}{c}{ Covariates } & Odds ratio $(\mathbf{9 5} \%$ CI) & $\boldsymbol{P}$ value \\
\hline Age (increasing, per year) & $1.05(1.03-1.07)$ & $<.001$ \\
Female sex & $0.96(0.66-1.39)$ & .81 \\
Comorbidities & & \\
$\quad$ Myocardial infarction & $1.90(1.08-3.36)$ & .03 \\
Congestive heart failure & $1.03(0.62-1.71)$ & .91 \\
Cerebrovascular disease & $4.92(3.40-7.11)$ & $<.001$ \\
COPD & $1.22(0.87-1.72)$ & .26 \\
$\quad$ Diabetes mellitus & $0.71(0.38-1.33)$ & .29 \\
Liver disease & $4.22(1.35-13.2)$ & .01 \\
$\quad$ Chronic renal insufficiency & $1.92(1.46-2.53)$ & $<.001$ \\
Nonteaching hospital & $1.06(0.70-1.62)$ & .78 \\
Rural hospital & $1.47(0.40-5.38)$ & .56 \\
Small or medium hospital & $1.29(0.86-1.96)$ & .22 \\
Early era & $2.12(1.28-3.50)$ & .003 \\
Open approach & $2.94(1.67-5.16)$ & $<.001$ \\
\hline DTAA, Descending thoracic aortic aneurysm; $C I$, confidence interval; COPD, chronic \\
obstructive pulmonary disease. & &
\end{tabular}

analysis, in which the overall operative mortality rates for open repair of intact and ruptured DTAAs were $6.3 \%$ and $36.6 \%$, respectively.

Data for TEVAR in the management of DTAA have been promising. A single-institution analysis of 224 patients undergoing TEVAR for DTAA demonstrated a 30-day mortality of $3 \%$ and a 1 -year mortality of $15 \% .{ }^{15}$ An analysis comparing TEVAR with open surgical repair at a single center demonstrated reduced 30 -day $(5.7 \%$ vs $25 \%)$ and 1 -year $(19 \%$ vs $42 \%)$ mortality rates with TEVAR. ${ }^{16}$

Multicenter trial data of 142 patients undergoing TEVAR demonstrated successful implantation of the device in $98 \%$, with a 30-day mortality of $1.5 \% .{ }^{17}$ A comparative study of TEVAR trial patients and an open surgical control cohort demonstrated reduced perioperative mortality $(2.1 \%$ vs $11.7 \%$ ), with lower rates of postoperative spinal cord ischemia, respiratory tract failure, and renal insufficiency in the TEVAR group. ${ }^{18}$ In The Valiant Thoracic Stent Graft System Clinical Study (VALOR II) trial, 160 patients underwent TEVAR for DTAA, with successful deployment in $96.3 \%$ and a perioperative mortality rate of $3.1 \% .{ }^{19}$

In addition to single-institutional and trial data, there have been prior analyses of nationwide outcomes, although none have examined repairs for both intact and ruptured DTAAs for all adult ages during an 11-year period, as our study did. In 1 analysis of NIS data between 2006 and 2007, TEVAR was associated with improved inpatient outcomes for intact DTAA repair. ${ }^{20}$ Another nationwide analysis of ruptured DTAA repair between 2006 and 2008 by the same group demonstrated equivalent outcomes between TEVAR and open repair. ${ }^{21}$ The discrepancy between the latter study and our study regarding the mortality benefit of TEVAR for ruptured DTAA may be
TABLE 4. Multivariable analysis for inpatient mortality after repair of ruptured DTAA

\begin{tabular}{lcc}
\hline \multicolumn{1}{c}{ Covariates } & Odds ratio $(\mathbf{9 5} \% \mathbf{C I})$ & $\boldsymbol{P}$ value \\
\hline Age (increasing, per year) & $1.03(1.01-1.05)$ & .001 \\
Female sex & $0.80(0.51-1.25)$ & .33 \\
Comorbidities & & \\
$\quad$ Myocardial infarction & $1.38(0.72-2.65)$ & .33 \\
Congestive heart failure & $0.94(0.55-1.62)$ & .83 \\
Cerebrovascular disease & $1.33(0.80-2.19)$ & .27 \\
COPD & $1.27(0.83-1.94)$ & .26 \\
Diabetes mellitus & $1.40(0.70-2.81)$ & .34 \\
Liver disease & $3.35(0.39-29.0)$ & .27 \\
Chronic renal insufficiency & $1.31(0.94-1.83)$ & .11 \\
Nonteaching hospital & $0.92(0.56-1.53)$ & .75 \\
Rural hospital & $1.88(0.02-163.2)$ & .78 \\
Small or medium hospital & $1.17(0.72-1.88)$ & .53 \\
Early era & $1.00(0.63-1.61)$ & .99 \\
Open approach & $2.72(1.47-5.02)$ & .001 \\
\hline
\end{tabular}

$D T A A$, Descending thoracic aortic aneurysm; $C I$, confidence interval; $C O P D$, chronic obstructive pulmonary disease.

because our analysis had more than 3-fold more patients and was adequately powered to detect a difference.

\section{Study Implications}

Our study adds to the literature on DTAA repair by providing a decade-long analysis of "real-world" data. Moreover, the NIS registry is a better reflection of realworld clinical practices and outcomes, compared with clinical registries or trials in which participation is voluntary and reporting biases are often present. Our analysis demonstrates that the findings from prior single-institution studies and trial data are generally mirrored on a national level, where TEVAR is associated with reduced riskadjusted operative mortality for repair of both intact and ruptured DTAAs. Perhaps most important, it demonstrates that more patients are able to undergo surgical intervention for this disease entity and that national outcomes have improved.

Despite these collective data demonstrating the shortterm advantages of an endovascular approach, the longerterm benefits of TEVAR remain to be established. Indeed, prior analyses of Medicare data have suggested otherwise. A study of Medicare patients undergoing DTAA repair from 2004 to 2007 demonstrated significant reductions in perioperative mortality with TEVAR, but equivalent 5-year survival between approaches. ${ }^{22}$ Another analysis of Medicare data demonstrated lower perioperative mortality but worse 1 - and 5-year survival with TEVAR compared with open DTAA repair. ${ }^{23}$ It is certainly plausible that the relative benefits of TEVAR lessen as the time from surgery lengthens, especially given the higher comorbidity burdens of the patients in this cohort. Close continued surveillance is a key component of any endovascular program because some patients may become 
lost to follow-up and risk rupture from a late endoleak. In addition, open surgical repair will remain an important component of DTAA treatment because some aneurysms are not amenable to endovascular approaches and some institutions or providers do not have the resources or training to perform TEVAR.

\section{Study Limitations}

A principal limitation of our analysis is the lack of radiologic data within the NIS registry. Accordingly, aneurysmal features that would be expected to affect outcomes, such as size, rate of progression, relation to branch vessels, and overall amenability to an endovascular approach, were not available. Other variables, including surgeon expertise and training and institutional resources, were not included in the analysis. Finally, our analysis was limited to inpatient outcomes, but longitudinal data, including long-term survival, functional recovery, and rates of endoleak for TEVAR, are additional end points that are relevant.

\section{CONCLUSIONS}

This population-based analysis of more than 20,000 DTAA repairs in the United States provides encouraging data, demonstrating that operative outcomes have improved during the past decade. In addition, the increasing use of TEVAR has allowed sicker patients to undergo surgery for DTAA, and has contributed to the overall increase in repair rates observed for both intact and ruptured cases. These data collectively support broader implementation and enablement of TEVAR for the management of DTAA.

\section{References}

1. Isselbacher EM. Thoracic and abdominal aortic aneurysms. Circulation. 2005; 111:816-28.

2. Bickerstaff LK, Pairolero PC, Hollier LH, Melton LJ, Van Peenen HJ, Cherry KJ, et al. Thoracic aortic aneurysms: a population-based study. Surgery. 1982;92:1103-8.

3. Conrad MF, Cambria RP. Contemporary management of descending thoracic and thoracoabdominal aortic aneurysms: endovascular versus open. Circulation. 2008; 117:841-52.

4. Nienaber CA, Kische S, Skriabina V, Ince H. Noninvasive imaging approaches to evaluate the patient with known or suspected aortic disease. Circ Cardiovasc Imaging. 2009;2:499-506.

5. Cambria RP, Davison JK, Zannetti S, L'Italien G, Brewster DC, Gertler JP, et al. Clinical experience with epidural cooling for spinal cord protection during thoracic and thoracoabdominal aneurysm repair. J Vasc Surg. 1997;25:234-41.

6. Coselli JS, Lemaire SA, Koksoy C, Schmittling ZC, Curling PE. Cerebrospinal fluid drainage reduces paraplegia after thoracoabdominal aortic aneurysm repair: results of a randomized clinical trial. J Vasc Surg. 2002;35:631-9.
7. Estrera AL, Miller CC 3rd, Chen EP, Meada R, Torres RH, Porat EE, et al. Descending thoracic aortic aneurysm repair: 12-year experience using distal aortic perfusion and cerebrospinal fluid drainage. Ann Thorac Surg. 2005;80: 1290-6.

8. Healthcare Cost and Utilization Project. Overview of the nationwide inpatient sample. Available at: http://www.hcup-us.ahrq.gov/nisoverview.jsp. Accessed January 27, 2013.

9. Charlson ME, Pompei P, Ales KL, MacKenzie CR. A new method of classifying prognostic comorbidity in longitudinal studies: development and validation. J Chronic Dis. 1987;40:373-83.

10. United States Census Bureau. US Census Bureau population estimates. Available at: http://www.census.gov/popest. Accessed January 27, 2013.

11. Rigberg DA, McGory ML, Zingmond DS, Maggard MA, Agustin M, Lawrence PF, et al. Thirty-day mortality statistics underestimate the risk of repair of thoracoabdominal aortic aneurysms: a statewide experience. J Vasc Surg. 2006;43:217-22.

12. Rigberg DA, Zingmond DS, McGory ML, Maggard MA, Agustin M, Lawrence PF, et al. Age stratified, perioperative, and one-year mortality after abdominal aortic aneurysm repair: a statewide experience. J Vasc Surg. 2006; 43:224-9.

13. Coselli JS, LeMaire SA, Conklin LD, Adams GJ. Left heart bypass during descending thoracic aortic aneurysm repair does not reduce the incidence of paraplegia. Ann Thorac Surg. 2004;77:1298-303.

14. Verdant A, Cossette R, Page A, Baillot R, Dontigny L, Page P. Aneurysms of the descending thoracic aorta: three hundred sixty-six consecutive cases resected without paraplegia. J Vasc Surg. 1995;21:385-90.

15. Scali ST, Chang CK, Feezor RJ, Hess PJ Jr, Beaver TM, Martin TD, et al. Preoperative prediction of mortality within 1 year after elective thoracic endovascular aortic aneurysm repair. J Vasc Surg. 2012;56:1266-73.

16. Andrassy J, Wiedenhagen R, Meimarakis G, Rentsch M, Jauch KW, Kopp R. Endovascular versus open treatment of degenerative aneurysms of the descending thoracic aorta: a single center experience. Vascular. 2011;19:8-14.

17. Makaroun MS, Dillavou ED, Kee ST, Sicard G, Chaikof E, Bavaria J, et al. Endovascular treatment of thoracic aortic aneurysms: results of the phase II multicenter trial of the GORE TAG thoracic endoprosthesis. J Vasc Surg. 2005;41:1-9.

18. Bavaria JE, Appoo JJ, Makaroun MS, Verter J, Yu ZF, Mitchell RS; Gore TAG Investigators. Endovascular stent grafting versus open surgical repair of descending thoracic aortic aneurysms in low-risk patients: a multicenter comparative trial. J Thorac Cardiovasc Surg. 2007;133:369-77.

19. Fairman RM, Tuchek JM, Lee WA, Kasirajan K, White R, Mehta M, et al; VALOR II Investigators. Pivotal results for the Medtronic Valiant Thoracic Stent Graft System in the VALOR II trial. J Vasc Surg. 2012;56:1222-31.

20. Gopaldas RR, Huh J, Dao TK, LeMaire SA, Chu D, Bakaeen FG, et al. Superior nationwide outcomes of endovascular versus open repair for isolated descending thoracic aortic aneurysm in 11,669 patients. J Thorac Cardiovasc Surg. 2010; 140:1001-10.

21. Gopaldas RR, Dao TK, LeMaire SA, Huh J, Coselli JS. Endovascular versus open repair of ruptured descending thoracic aortic aneurysms: a nationwide risk-adjusted study of 923 patients. J Thorac Cardiovasc Surg. 2011;142: 1010-8.

22. Conrad MF, Ergul EA, Patel VI, Parachuri V, Kwolek CJ, Cambria RP. Management of diseases of the descending thoracic aorta in the endovascular era: a Medicare population study. Ann Surg. 2010;252:603-10.

23. Goodney PP, Travias L, Lucas FL, Fillinger MF, Goodman GC, Cronenwett JL, et al. Survival after open versus endovascular thoracic aortic aneurysm repair in an observational study of the Medicare population. Circulation. 2011;124: 2661-9. 